\title{
Internal Model Control for Singular Multivariable Systems Based on Kronecker Canonical Form
}

\author{
Qibing Jin, Qi Wang*, Beiyan Jiang, Meiying Jiang and Yan Huang \\ Institute of Automation \\ Beijing University of Chemical Technology \\ Beisanhuan East Road 15, Chaoyang District, Beijing, PR China \\ *Corresponding author
}

\begin{abstract}
The internal model control design method for stable plants has enjoyed much popularity due to its simple yet effective procedure. In this paper, a robust IMC method is proposed for multivariate singular systems on the basis of Kronecker canonical form, which allows us to analysis and control of a singular linear system conveniently. Instead of considering the state space approach, the proposed procedure is derived on the basis of transfer function matrix. Based on this equivalent restrict decomposition form, the control object is first decomposed into separate slow and fast subsystems of lower dimensions and permitted a simple design. Then a feed forward controller is designed to eliminate impulse for fast subsystem, followed by that an internal model controller is designed for slow subsystem. For ease of implementation's sake, time-scale transformation is introduced. The proposed method is also verified applicable for impulse-free singular systems. In order to improve control precisions, the diagonal filter is designed with Genetic algorithms. During the optimization process, the integral of squared error index is adopted. Simulation results demonstrate the validity of the proposed approaches.
\end{abstract}

Keywords- multivariate singular system; internal model control; transfer function; kronecker canonical form

\section{INTRODUCTION}

In the engineering practice, we often encounter a class of dynamic systems that its plant model cannot be described by ordinary differential equations for the relationships of some physical variables are dynamic while others purely static. Fortunately, singular system models offer a more effective way to describe these dynamic systems than the regular ones for containing a mixture of algebraic and differential equations. Singular systems have been widely studied over recent decades due to their capacity to describe non-dynamic constraints and impulsive behaviors. And a large number of fundamental concepts and results based on the theory of the normal state-space systems have been extended successfully to singular systems during the past decades. For example, act as one of the most important and basic properties for singular linear systems, regularization have been studied by many researchers, e.g. [1]. Other basic problems, which are reflected in the topics of stabilization, optimal control, impulse elimination, and observer design for singular systems have been extensively studied by numerous scholars[2-5].

In addition to these, other control problems in singular linear systems such as $H_{\infty}$ control, fault-tolerant control, and filtering have been analyzed by a considerable authors; see, e.g., [6]-[8], and the reference therein. Furthermore, these problems and corresponding results have been extended to nonlinear singular systems and some more complicated singular linear systems; see, e.g., [9]-[10]. Moreover, as an important part in the general field of control systems theory, singular system theoryhas been used as a tool to treat the design problems of some more complicated normal systems for their convenient representation of real systems; for example, [11] considered $H_{\infty}$ control using singular system approach and [12] introduced a singular system approach to investigate robust fault detection for Takagi-Sugeno models.

On the other hand, there have been great efforts dedicated to the study of internal model control (IMC) because of its simple structure and fewer parameters to be tuned on-line. As a particular control structure, IMC has been widely employed in process control and obtained successful applications in industries in the last twenty years or so; see [13]-[14]. However, most of the researches about IMC are specific to those systems whose transfer functions are proper or strictly proper. For singular systems, there are very few works on IMC has been reported. Therefore, constructing rational control structures and extending IMC method to singular systems truly possess of both theoretical interest and practical significance.

In this paper, we attempt to adopt IMC to study the robust control problem of singular systems based on transfer function matrix. To this end, the given system is first decomposed into slow and fast subsystems by Kronecker canonical form. Then the control problem is converted into design the controllers for the slow and the fast subsystems. A feed forward controller is designed to eliminate impulse for fast subsystem, followed by that an internal model controller is designed for slow subsystem. Furthermore, the proposed method is verified applicable for impulse-free singular systems. In order to improve control precisions, the diagonal filter is designed with Genetic algorithms. During the optimization process, the integral of squared error index is adopted. Finally, an example is adopted to demonstrate the validity and feasibility of the new method. It is shown that the proposed strategy not only guarantees control effect of the closed-loop system, but also simultaneously eliminates the impulsive behavior of the fast subsystem.

The layout of the paper is the following. The class of 
studied systems and some definitions and major limitations are presented in Section II, as well as the basic principle of IMC. Section III devotes to the proposed method and design procedure of corresponding controllers while Section IV discusses the applied object of the presented method. In Section V, the example and simulations are given to examine the algorithm both in terms of robustness properties and closed loop performance. Finally, the concluding remarks are given in Section VI.

\section{PROBLEM STATEMENT AND PRELIMINARIES}

It is well-known that a singular system of the form

$$
\left\{\begin{array}{l}
\boldsymbol{E} \dot{\boldsymbol{x}}(t)=\boldsymbol{A} \boldsymbol{x}(t)+\boldsymbol{B} \boldsymbol{u}(t)+\boldsymbol{B}_{1} \boldsymbol{w}(t) \\
\boldsymbol{y}(t)=\boldsymbol{C} \boldsymbol{x}(t)
\end{array}\right.
$$

where $\boldsymbol{x}(t) \in R^{n}, \boldsymbol{u}(t) \in R^{m}, \boldsymbol{w}(t)$ and $\boldsymbol{y}(t) \in R^{p}$ are, respectively, the state vector, the input vector, the disturbance vector and the output vector; $\boldsymbol{A} \in R^{n \times n}, \boldsymbol{B} \in R^{n \times m}, \boldsymbol{B}_{1} \in R^{n \times m}$ and $\boldsymbol{C} \in R^{p \times n}$, $(m, p<n)$ are known nonsingular constant matrices. The matrix $\boldsymbol{E} \in R^{n \times n}$ may be singular with $\operatorname{rank}(\boldsymbol{E})=n_{1}<n$. To guarantee that system (1) has an unique solution, we hereafter assume that matrix pair $(\boldsymbol{E}, \boldsymbol{A})$ is regular, $i, e$. $\exists s \in \boldsymbol{C}, \operatorname{det}(s \boldsymbol{E}-\boldsymbol{A}) \neq 0$. By Laplace transformation, the transfer function matrix of system (1) is obtained as follows:

$$
\boldsymbol{G}(s)=\boldsymbol{C}(s \boldsymbol{E}-\boldsymbol{A})^{-1} \boldsymbol{B}
$$

The purpose of this subsection is to outline the basic problems for descriptor linear systems analysis and design. For the convenience of discussion, we state some related definitions and results which are all equivalent to the original definitions.

\section{Definition $1^{[15]}$ :}

1) Consider singular system (1), matrix pair $(\boldsymbol{E}, \boldsymbol{A})$ is regular if there exists a constant $\alpha \in \mathrm{C}$ such that $\operatorname{det}(\alpha \boldsymbol{E}-\boldsymbol{A}) \neq 0$, or the polynomial $\operatorname{det}(s \boldsymbol{E}-\boldsymbol{A})$ is not identically zero for $s \in \boldsymbol{C}$;

2) Consider singular system (1), matrix pair $(\boldsymbol{E}, \boldsymbol{A})$ is said to be stable if $\sigma(\boldsymbol{E}, \boldsymbol{A}) \in\{\mathrm{s} \in \mathrm{C} \mid \operatorname{det}(\mathrm{s} \boldsymbol{E}-\boldsymbol{A})=0\} \subset \mathrm{C}^{-}$

3) Consider Singular system (1), matrix pair $(\boldsymbol{E}, \boldsymbol{A})$ is impulse-free if $\operatorname{deg}\{\operatorname{det}(s \boldsymbol{E}-\boldsymbol{A})\}=\operatorname{rank}(\boldsymbol{E})$.

4) Singular system (1) is admissible if it is regular, stable and impulse-free.

Admissibility is the essential condition for ensuring the running of generalized system normally. Usually, the actual system is essentially useless if it doesn't meet the requirement of admissibility. For a properly stabilizable and properly detectable singular system (1), the internal properness and stability are equivalent to the external properness and stability. So in this paper, we assume system (1) is a completely controllable, properly detectable, and stable plant.

It is known from [15] that there exist a state transformation
$\boldsymbol{P}_{2}^{-1} \boldsymbol{x}=\left[\begin{array}{ll}\boldsymbol{x}_{1}^{T} & \boldsymbol{x}_{2}^{T}\end{array}\right]^{T}$ and two nonsingular matrices $\boldsymbol{P}_{1}, \boldsymbol{P}_{2}$ such that system (1) is transformed into the following equivalent standard decomposition form:

$$
\begin{aligned}
& \dot{x}_{1}(t)=A_{1} x_{1}(t)+B_{1} u(t), \quad y_{1}(t)=C_{1} x_{1}(t) \\
& N \dot{x}_{2}(t)=x_{2}(t)+B_{2} u(t), \quad y_{2}(t)=C_{2} x_{2}(t)
\end{aligned}
$$

$$
\boldsymbol{y}(t)=\boldsymbol{y}_{1}(t)+\boldsymbol{y}_{2}(t)
$$

where, $\quad \boldsymbol{x}_{1}(t) \in \boldsymbol{R}^{n_{1}}, \quad \boldsymbol{x}_{2}(t) \in \boldsymbol{R}^{n_{2}}, \quad n_{1}+n_{2}=n, \quad \boldsymbol{N} \in \boldsymbol{R}^{n_{2} \times n_{2}} \quad$ is nilpotent whose nilpotent denoted by $h$, if $\boldsymbol{N}^{h-1} \neq 0$ and $\boldsymbol{N}^{h}=0$

Then the transfer function matrix of system (1) can be expressed as

$$
\begin{aligned}
\boldsymbol{G}(s)= & \boldsymbol{C}(s \boldsymbol{E}-\boldsymbol{A})^{-1} \boldsymbol{B}=\boldsymbol{C}_{1}\left(s \boldsymbol{I}-\boldsymbol{A}_{1}\right)^{-1} \boldsymbol{B}_{1} \\
& +\boldsymbol{C}_{2}(s \boldsymbol{N}-\boldsymbol{I})^{-1} \boldsymbol{B}_{2}=\boldsymbol{G}_{s}(s)+\boldsymbol{G}_{q}(s)
\end{aligned}
$$

From formula (4), we can see that, the transfer function matrix of system (1) consists of two parts: one is $\boldsymbol{G}_{s}(s)$, which is determined by the slow subsystem and is strictly proper; the other is a polynomial $\boldsymbol{G}_{q}(s)$, which is determined by the fast subsystem. For convenience, we often call the transfer function matrix $\boldsymbol{G}_{s}(s)$ the slow subsystem, and polynomial $\boldsymbol{G}_{q}(s)$ the fast subsystem.

\section{CONTROLLER DESIGN SCHEM}

In Section II, we have known that singular system (1) can be equivalent to the sum of slow subsystem (3-a) and fast subsystem (3-b) based on the standard decomposition form. Then the control problems of singular linear system (1) is converted into that of the slow and the fast subsystems. The improved IMC design scheme used in the system is illustrated on Figure 1.

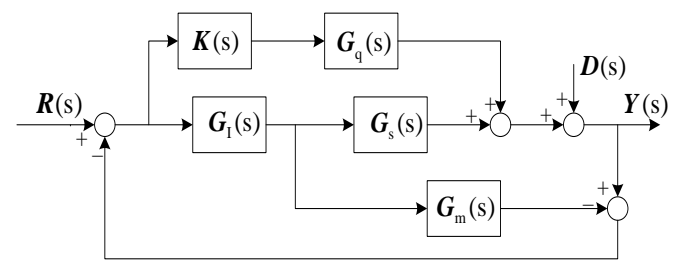

FIGURE I. THE CONTROL STRUCTURE OF SINGULAR SYSTEM

From Figure 1 we can found that the relation of input, output and disturbance as:

$$
\boldsymbol{Y}(s)=\boldsymbol{G}_{R Y}(s) \boldsymbol{R}(s)+\boldsymbol{G}_{D Y}(s) \boldsymbol{D}(s)
$$

where, 


$$
\begin{aligned}
\boldsymbol{G}_{R Y}(s) & =\frac{\boldsymbol{G}_{s}(s) \boldsymbol{G}_{I}(s)+\boldsymbol{G}_{q}(s) \boldsymbol{K}(s)}{\boldsymbol{I}+\left[\boldsymbol{G}_{s}(s)-\boldsymbol{G}_{m}(s)\right] \boldsymbol{G}_{I}(s)+\boldsymbol{G}_{q}(s) \boldsymbol{K}(s)} \\
\boldsymbol{G}_{D Y}(s) & =\frac{\boldsymbol{I}-\boldsymbol{G}_{m}(s) \boldsymbol{G}_{I}(s)}{\boldsymbol{I}+\left[\boldsymbol{G}_{s}(s)-\boldsymbol{G}_{m}(s)\right] \boldsymbol{G}_{I}(s)}
\end{aligned}
$$

In this scheme, the dynamic compensator $\boldsymbol{K}(s)$ is proposed to address the impulse elimination problem of $\boldsymbol{G}_{q}(s)$. The internal model controller $\boldsymbol{G}_{\mathrm{I}}(s)$, which is designed based on the approximate mode $\boldsymbol{G}_{m}(s)$, is used to suppress disturbance since $\boldsymbol{G}_{s}(s)$ is nothing but that of a normal linear system.

\section{A. IMC Controller}

Suppose $\boldsymbol{G}_{s}(s)=\boldsymbol{G}_{m}(s)$, the internal model controller $\boldsymbol{G}_{\mathrm{I}}(s)$ is designed as

$$
\boldsymbol{G}_{I}(s)=\boldsymbol{G}_{m}^{-1}(s) \boldsymbol{G}_{m+}(s) \boldsymbol{F}(s)
$$

where, $\boldsymbol{F}(s)=\operatorname{diag}\left\{1 /\left(1+\lambda_{i} s\right)^{n_{i}}\right\}, i=1,2, \cdots, p$ is a low-frequency filter to ensure the realization of $\boldsymbol{G}_{\mathrm{I}}(s)$ and attenuate the effects of process model mismatch. The filter order $n_{i}$ is selected sufficiently large to guarantee $\boldsymbol{G}_{\mathrm{I}}(s)$ proper, to prevent excessive differential control action, while $\lambda_{i}$ is an adjustable parameter which determines the speed of response. $\boldsymbol{G}_{m+}(s)$ is non-minimum-phase, which contains time delay in the stable process and right half plane zeros. Letting $\boldsymbol{G}_{m+}(s)$ has the form

$$
\boldsymbol{G}_{m+}(s)=\operatorname{diag}\left\{e^{-\theta_{K_{i}} s} \prod_{1}^{D_{i}}\left(\frac{-\mathrm{s}+z_{p}}{s+z_{p}^{*}}\right)^{W_{i}}\right\}, \quad i=1,2, \cdots, p
$$

where $\theta_{K i}$ is the maximum predictive term in the $i$-th column of $\boldsymbol{G}_{m}^{-1}(s) ; z_{p}$ is the right half-plane poles in corresponding column; $z_{p}^{*}$ is the conjugate complex of $z_{p} ; W_{i}$ represents number of the same poles in the $i$-th column of $\boldsymbol{G}_{m}^{-1}(s) ; D_{i}$ indicates the number of distinct poles exist in the $i$-th column of $\boldsymbol{G}_{m}^{-1}(s)$.

Substitute formula into formula (5), yields

$$
\boldsymbol{Y}(s)=\frac{\boldsymbol{G}_{m+}(s) \boldsymbol{F}(s)+\boldsymbol{G}_{q}(s) \boldsymbol{K}(s)}{\boldsymbol{I}+\boldsymbol{G}_{q}(s) \boldsymbol{K}(s)} \boldsymbol{R}(s)+\left(\boldsymbol{I}-\boldsymbol{G}_{m+}(s) \boldsymbol{F}(s)\right) \boldsymbol{D}(s)
$$

\section{B. Elimination of Impulses by Dynamic Compensator}

All It is clear that, the internal model controller can resist disturbance. Next, we discuss the design method of controller $\boldsymbol{K}(s)$ by analyzing the zero steady-state error characteristic for the closed-loop system.

From formula (8), the closed-loop error equation of IMC is:

$$
\begin{aligned}
\boldsymbol{E}(s)= & \boldsymbol{R}(s)-\boldsymbol{Y}(s)=\frac{\boldsymbol{I}-\boldsymbol{G}_{m+}(s) \boldsymbol{F}(s)}{\boldsymbol{I}+\boldsymbol{G}_{q}(s) \boldsymbol{K}(s)} \boldsymbol{R}(s) \\
& -\left(\boldsymbol{I}-\boldsymbol{G}_{m+}(s) \boldsymbol{F}(s)\right) \boldsymbol{D}(s)
\end{aligned}
$$

By analyzing the previous controller design method, we know that $\boldsymbol{G}_{m+}(0) \boldsymbol{F}(0)=\boldsymbol{I}$. When step input and disturbance input acting on the system, the steady-state error is:

$$
e(\infty)=\lim _{s \rightarrow 0} s \boldsymbol{E}(s)=0
$$

Formula (10) indicates that the controller $\boldsymbol{K}(s)$ contributes nothing to system zero steady-state error characteristic. So the form of the controller can be designed as simply as possible under the premises of eliminating impulse, realizing decoupling system, ensuring the system stability and possible responding speed.

From the preceding description, we know that the transfer function of $\boldsymbol{G}_{q}(s)$ is a matrix polynomials, which means that $\boldsymbol{G}_{q}(s)$ is not proper. Take the time-scale transformations $\tau=\frac{1}{s}$, then $\boldsymbol{G}_{q}(s)$ is easily seen to generate the same transfer function

$$
\overline{\boldsymbol{G}}_{q}(s)=-\frac{1}{s} \boldsymbol{G}_{q}\left(\frac{1}{s}\right)
$$

By several transformations, $\overline{\boldsymbol{G}}_{q}(s)$ is illustrated to be strictly proper, which may be viewed as the transfer function matrix of a normal system. It is easy to verify that the order of molecular is no larger than that of denominator. [9] shows that the closed-loop system can be made impulse-free by almost any output feedback if and only if $\boldsymbol{G}_{q}(s)$ is both impulse controllable and observable. To keep the order of the compensator $\boldsymbol{K}(s)$ low, the designer should choose a reasonable feedback controller such that $\boldsymbol{K}(s)$ as simple as possible under the condition of the closed-loop subsystem is stable. Suppose that $\overline{\boldsymbol{G}}_{q}(s)$ has no right half plane zero, Select $\boldsymbol{K}(s)$ as

$$
\boldsymbol{K}(s)=\overline{\boldsymbol{G}}_{q}^{-1}(0) \boldsymbol{F}_{q}(s)
$$

Where, $\boldsymbol{F}_{q}(s)=\operatorname{diag}\left\{(s+1)^{-1}\right\}$ is used to eliminate algebraic loop, $\overline{\boldsymbol{G}}_{q}^{-1}(0)$ is selected to ensure the subsystem can realize static decoupling.

The main idea of the method is trying to design a controller $\boldsymbol{K}(s)$ such that the fast system $\boldsymbol{G}_{q}(s)$ is admissible. Furthermore, the incorporation in the feed-forward path can be regarded as the disturbance which is relevant with the set point input. It has been proved that IMC can achieves disturbance rejection in spite of the existing of model error.

\section{SOME DISCUSSIONS OF THE PROPOSED}

After the text edit has been completed, the paper is ready for the template. Duplicate the template file by using the Save 
As command, and use the naming convention prescribed by your conference for the name of your paper. In this newly created file, highlight all of the contents and import your prepared text file. You are now ready to style your paper; use the scroll down window on the left of the MS Word Formatting toolbar.

\section{A. The Discussion of Controlled Plant}

In this paper, we assume that the system (1) contains some impulses which result in the transfer function $\boldsymbol{G}(s)$ is not a strictly proper rational matrix. This method can be extended to impulse-free singular systems. If the system is impulse-free, that is to say, in formula (3-b), $N=0$. Then system (1) is reduced to a normal dynamic system, and the corresponding transfer function is

$$
\overline{\boldsymbol{G}}(s)=\boldsymbol{C}(s \boldsymbol{E}-\boldsymbol{A})^{-1} \boldsymbol{B}+\boldsymbol{D}=\boldsymbol{C}_{1}\left(s \boldsymbol{I}-\boldsymbol{A}_{1}\right)^{-1} \boldsymbol{B}_{1}+\left(-\boldsymbol{C}_{2} \boldsymbol{B}_{2}\right)
$$

It is not hard to see that $\overline{\boldsymbol{G}}(s)$ is regular. Therefore, under the condition of $\boldsymbol{N}=0$, the system (1) can be controlled using any conventional technique. In other words, we can use the abundant results in linear system theory to study it.

\section{B. The Discussion of Controller Optimization}

Since the proper controller response to both regulatory control and servo control is determined by finding reasonable valves of corresponding controller parameters, the optimization of controllers parameters should always be taken into account when designing controllers. Genetic algorithms (GAs) [16] characterized by high parallelism and good overall situation astringency have been proven to be an effective method in solving the optimal problems by previous work. On the other hand, to comprehensively evaluate the dynamic performance of closed-loop system, the integral of squared error index (ISE)[17] which considers the sum of the ISE values in response and interaction, has been widely taken as the performance index.

In order to improve the control performance and avoid a great deal of adjusting work of the parameters, the GAs method is adopted in design of the controllers based on taking ISE criterion as the performance index.

\section{Simulation}

Consider the non-proper transfer-function matrix[5]

$$
\boldsymbol{G}(s)=\left[\begin{array}{cc}
\frac{s+2}{s+1} & \frac{1}{s+2} \\
\frac{-s-1}{s+2} & \frac{s^{2}+3 s+3}{s+1}
\end{array}\right]
$$

The transfer functions of slow sub-system $\boldsymbol{G}_{s}(s)$ and fast sub-system $\boldsymbol{G}_{q}(s)$ are:

$$
\boldsymbol{G}_{s}(s)=\left[\begin{array}{cc}
\frac{1}{s+1} & \frac{1}{s+2} \\
\frac{1}{s+2} & \frac{1}{s+1}
\end{array}\right], \boldsymbol{G}_{q}(s)=\left[\begin{array}{cc}
1 & 0 \\
-1 & s+2
\end{array}\right]
$$

Then, the controllers are given as

$$
\begin{gathered}
\boldsymbol{G}_{I}(s)=\left[\begin{array}{cc}
\frac{(s+1)(s+2)^{2}}{\left(\lambda_{1} s+1\right)^{2}(2 s+3)} & \frac{(s+1)^{2}(s+2)}{\left(\lambda_{2} s+1\right)^{2}(2 s+3)} \\
\frac{(s+1)^{2}(s+2)}{\left(\lambda_{2} s+1\right)^{2}(2 s+3)} & \frac{(s+1)(s+2)^{2}}{\left(\lambda_{1} s+1\right)^{2}(2 s+3)}
\end{array}\right], \\
\boldsymbol{K}(s)=\frac{1}{s+1}\left[\begin{array}{cc}
k_{1} & 0 \\
0.5 & 0.5 k_{2}
\end{array}\right]
\end{gathered}
$$

For given the step input $r_{1}=1, r_{2}=2$, and disturbance input $d_{1}=-0.4, d_{2}=0.4$ the optimum parameters of digital controllers are found as $\lambda_{1}=2.1265, \lambda_{2}=1.3249, k_{1}=0.8216$, and $k_{2}=0.4500$.

The performance of the controllers for set point responses and disturbance rejection are evaluated. Simulations run address the set point tracking capabilities and interference resistance abilities for both model matching and parametric uncertainties. Beside these, error changes are also shown.

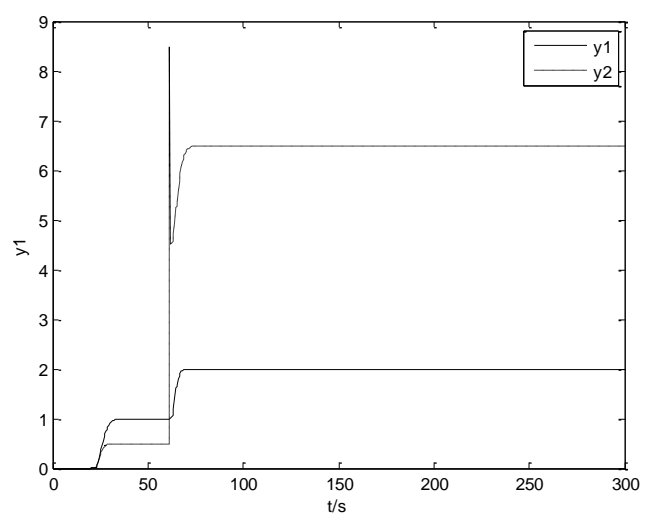

FIGURE II. OUTPUT RESPONSES OF UNCONTROLLED SYSTEM

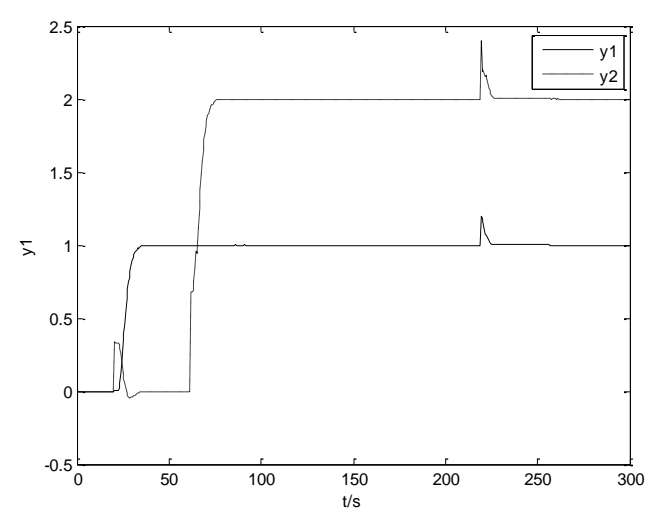

FIGURE III. OUTPUT RESPONSES OF CONTROLLED SYSTEM WHEN MODEL MATCHES COMPLETELY 


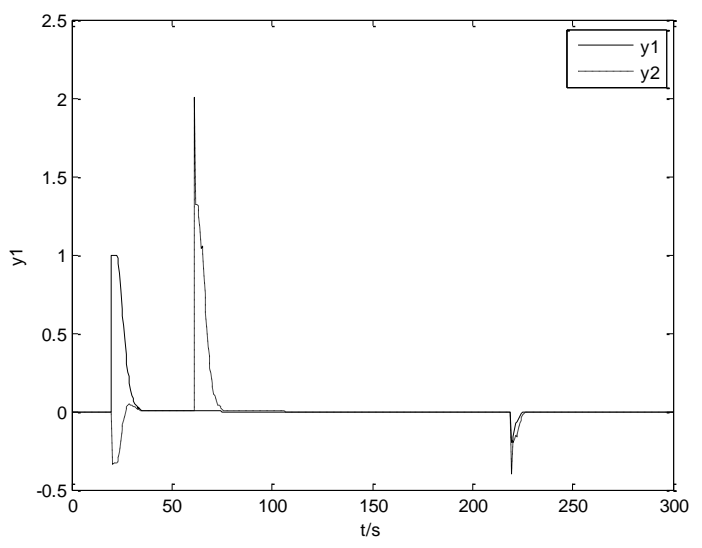

FIGURE IV. SYSTEM TRACK ERRORS

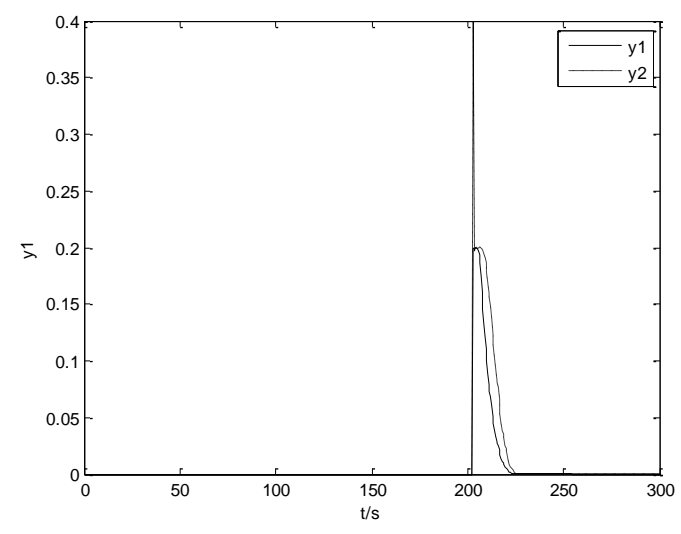

FIGURE V. DISTURBANCE RESPONSES

Analyzing from these figures, we can conclude that the proposed control scheme can follows the set valus and rejects step disturbance satisfactorily and system reaches desired values.

To validate the robustness of the proposed method, we increase the gains and time constants by $20 \%$ respectively when the controller settings remain the same as those provided for the perfect model matching. Figures 6 to 8 show the output responses of the system when the model mismatched.

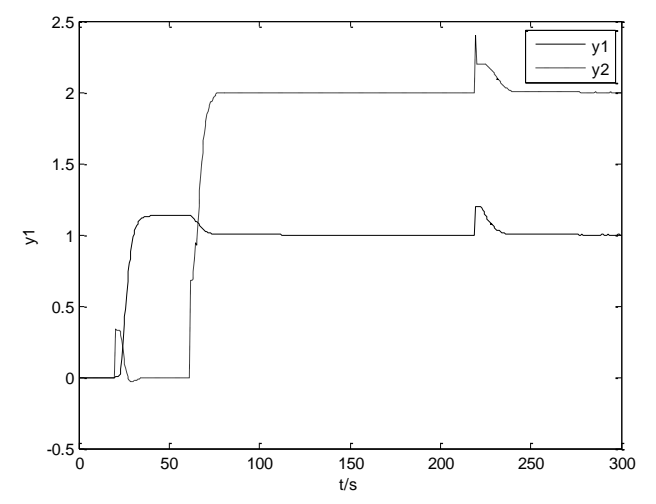

FIGURE VI. OUTPUT RESPONSES OF CLOSED-LOOP SYSTEM WHEN UNCERTAINTIES EXIST

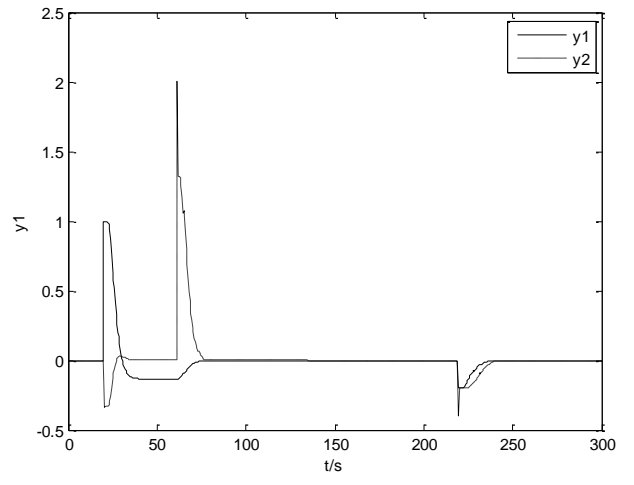

FIGURE VII. SYSTEM TRACK ERRORS WHEN UNCERTAINTIES EXIST

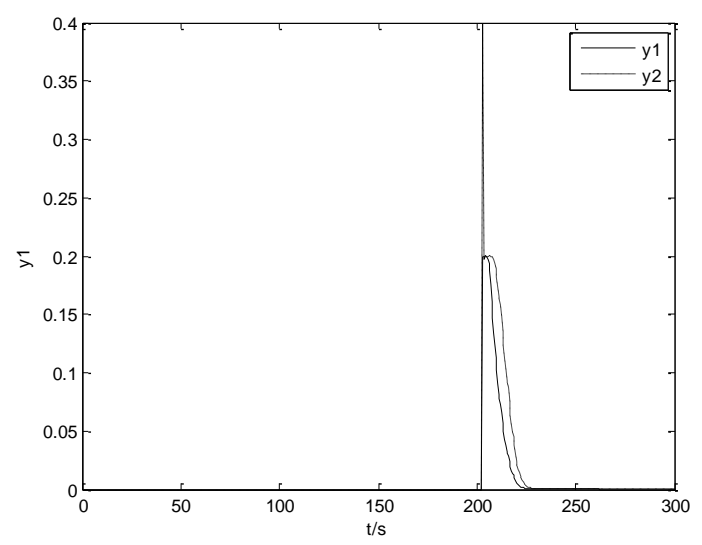

FIGURE VIII. DISTURBANCE RESPONSES WHEN UNCERTAINTIES EXIST

Judging from the simulated curves of these figures, we can conclude that the output responses obtained by the proposed method can follow the initial value with no steady error. Furthermore, the system still has good tracking performance and robustness in the presence of disturbance and uncertainties. Moreover, the designed controller is easy to compute and realize. In a word, the designed controller works well, and can achieve a certain control level.

\section{CONCLUSIONS}

In this paper, a robust IMC method was first proposed for multivariate Singular systems on the basis of Kronecker canonical form, which allows us to analyze and control of a singular linear system conveniently. Instead of considering the state space approach, the proposed procedure is derived on the basis of transfer function matrix. Based on this equivalent restrict decomposition form, the control object is first decomposed into separate slow and fast subsystems of lower dimensions and permitted a simple design. Then a feed forward controller is designed to eliminate impulse for fast subsystem, followed by that an internal model controller is designed for slow subsystem. For ease of implementation's sake, time-scale transformation is introduced. The proposed method is also verified applicable for impulse-free singular systems. In order to improve control precisions, the diagonal 
filter is designed with Genetic algorithms. During the optimization process, the integral of squared error index is adopted. Simulation results demonstrate the validity of the proposed approaches.

\section{ACKNOWLEDGMENT}

This work was partially supported by the financial support of the National Science Foundation of China (Grant 61273132) and the National Grand Fundamental Research 973 Program of China (Grant 2007CB714300) and partly by the Automation Institute Beijing University of Chemical Technology. The authors are grateful to the anonymous reviewers for their valuable recommendations.

\section{REFERENCES}

[1] A. Bunse-Gerstner, V. Mehrmann, and N. K. Nichols, "Regularization of singular systems by derivative and proportional state feedback," SIAM J. Matrix Anal. Appl., vol. 13, no. 1, pp. 46-67, Jul. 1992.

[2] E. L. Yip and R. F. Sincovec, "Solvability, controllability and observability of continuous singular systems," IEEE Trans. Automat. Contr., vol. 26, no. 3, pp. 702-706, Jun. 1981.

[3] H. Frankowska, "On controllability and observability of implicit systems," Syst. Control Lett., vol. 14, no. 3, pp. 219-225. Mar. 1990.

[4] G. R. Duan, "Eigenstructure assignment and response analysis in singular linear systems with state feedback control," Int. J. Control, vol. 69, no. 5, pp. 663-694, Nov. 1998.

[5] G. R. Duan, "Analysis and Design of Singular Linear Systems," New York: Springer-Verlag, 2010.

[6] A. Rehm and F. Allgöwer, "Self-scheduled $\mathrm{H} \infty$ output feedback control of singular systems," Comput. Chem. Eng., vol. 24, no. 2-7, pp. 279-284, Jul. 2000.

[7] Y. P. Zhang and J. T. Sun, "Impulsive robust fault-tolerant feedback control for chaotic Lur'e systems," Chaos, Solitons \& Fractals, vol. 39, no. 3, pp. 1440-1446, Feb. 2009.

[8] M. Ezzine, H. S. Ali, M. Darouach, and H. Messaoud, "A controller design based on a functional $\mathrm{H} \infty$ filter for singular systems: The time and frequency domain cases," Automatica, vol. 48, no. 3, pp. 542-549, Mar. 2012.

[9] H. Hamdi, M. Rodrigues, C. Mechmeche, and N. B. Braiek, "Robust Fault Detection and Estimation for Singular Systems based on MultiModels Concept," Int. J. Control Autom. Syst., vol. 10, no. 6, pp. 12601266, Dec. 2012.

[10] E. K. Boukas, S. Xu, and J. Lam, "On Stability and Stabilizability of Singular Stochastic Systems with Delays," J. Optimiz. Theory. App, vol. 127, no. 2, pp. 249-262, Nov. 2005.

[11] U. Shaked and E. Fridman, "A singular system approach to $\mathrm{H} \infty$ control of linear time-delay systems," IEEE Trans. Automat. Contr. , vol. 47, no. 2, pp. 253-270, 2002.

[12] M. Bouattour, M. Chadli, M. Chaabane, and A. E. Hajjaji, "Design of robust fault detection observer for Takagi-Sugeno models using the singular approach,” Int. J. Control Autom. Syst., vol. 9, no. 5, pp. 973 979, Oct. 2011.

[13] C. E. Garcia and M. Morari, "Internal Model Control. 1. A Unifying Review and Some New Result," Ind. Eng. Chem. Res. Design and Development, vol. 21, no. 2, pp. 308-323, 1982.

[14] M. M. Awais, "Application of internal model control methods to industrial combustion," Applied Soft Computing, vol. 5, no. 2, pp. $223-$ 233, Jan. 2005.

[15] G. R. Duan, "Analysis and Design of Singular Linear Systems," New York: Springer-Verlag, 2010.

[16] J. J. Grefenstette, "Optimization of control parameters for genetic algorithms. IEEE Trans. Syst. Man Cybern., vol. 16, no. 1, pp. 122-128, Jan. 1986.

[17] Q. B. Jin, F. Hao, and Q. Wang, "IMC-PID Controller Based on Diagonal Equivalent Model for Multivariable Systems with Multiple Time Delays," J. Chem. Eng. Jpn, vol. 46, no. 3, pp. 209-218, Mar. 2013. 